CLINICAL STUDY

\title{
The success rate of $I-131$ ablation in differentiated thyroid cancer: comparison of uptake-related and fixed-dose strategies
}

\author{
Robbert B T Verkooijen $^{1,2, *}$, Frederik A Verburg ${ }^{2, *}$, Johannes W van Isselt ${ }^{2}$, Cornelis J M Lips ${ }^{3}$, Jan W Smit ${ }^{4}$ \\ and Marcel P M Stokkel ${ }^{1}$ \\ ${ }^{1}$ Division of Nuclear Medicine, Department of Radiology, Leiden University Medical Center, C4-Q, Albinusdreef 2, PO Box 9600,2300 RC Leiden, The \\ Netherlands, Departments of ${ }^{2}$ Nuclear Medicine and ${ }^{3}$ Endocrinology, University Medical Center Utrecht, Utrecht, The Netherlands and ${ }^{4}$ Department of \\ Endocrinology and Metabolism, Leiden University Medical Center, Leiden, The Netherlands \\ (Correspondence should be addressed to M P M Stokkel; Email: m.p.m.stokkel@lumc.nl)
}

*R B T Verkooijen and F A Verburg contributed equally to this work

\begin{abstract}
Introduction: The aim of the study was to compare the success rate of an uptake-related ablation protocol in which the dose depends on an I-131 24-h neck uptake measurement and a fixed-dose ablation protocol in which the dose depends on tumour stage.

Methods: All differentiated thyroid carcinoma patients with M0 disease who had undergone (near-) total thyroidectomy followed by I-131 ablation were included. In the uptake-related ablation protocol, 1100 (uptake $>10 \%$ ), 1850 (uptake 5-10\%) and $2800 \mathrm{MBq}$ (uptake $<5 \%$ ) were used. In the fixeddosage ablation strategy, 3700 (T1-3, N0 stage) and $5550 \mathrm{MBq}$ (N1 and/or T4 stage) were applied. We used I-131 uptake on whole-body scintigraphy and thyroglobulin-off values to evaluate the ablation 6-12 months after treatment.

Results: In the uptake-related ablation protocol, 60 out of $139(43 \%)$ patients were successfully treated versus 111 out of 199 for the fixed-dose ablation protocol $(56 \%)(P=0.022)$. The differences were not statistically significant for patients with T4 $(P=0.581)$ and/or N1 $(P=0.08)$ disease or for patients with T4N1 tumour stage $(P=0.937)$.

Conclusion: The fixed-dose I-131 ablation protocol is more effective in ablation of the thyroid remnant in differentiated thyroid carcinoma patients than an uptake-related ablation protocol. This difference is not observed in patients with a N1 and/or T4 tumour stage.

European Journal of Endocrinology 159 301-307
\end{abstract}

\section{Introduction}

The therapy of choice in patients suffering from differentiated thyroid cancer (DTC), subdivided into papillary and follicular thyroid carcinoma, is (near-) total thyroidectomy. This is routinely followed by the administration of radioiodine-131 (I-131) to destroy any remaining benign or malignant thyroid tissue, so-called ablation. There are several reasons for routine ablation after surgery $(1,2)$ : a) to be able to detect a carcinoma recurrence by radioiodine scanning; b) radioiodine can destroy microscopic foci of carcinoma in the thyroid remnant; c) possible carcinoma outside the thyroid bed may be detected and treated by radioiodine and d) to use thyroglobulin $(\mathrm{Tg})$ as tumour marker of recurrent carcinoma all normal thyroid tissue has to be destroyed. Although I-131 has been used for many years to ablate thyroid remnants following thyroid surgery, a single optimal ablation strategy is still not established. Reports on the amount of I-131 required to achieve successful ablation show a considerable range (3-7).
At the Leiden University Medical Center (LUMC) and the University Medical Center Utrecht (UMCU), two academic hospitals in the Netherlands, different ablation strategies have been used over the years. At the LUMC, a relatively low-dose uptake-related ablation strategy was applied until June 2002 (8), whereas in the UMCU a fixed-dose strategy with relatively high administered I-131 activities has been in use since January $1990(9,10)$. The aim of this study was to compare the success rates of ablation according to these two above-mentioned protocols.

\section{Methods}

\section{Study population}

Patients were selected who received ablation treatment with I-131 at the LUMC or UMCU from January 1990, as this date marks the start of the fixed-dose protocol in the UMCU. Both subgroups consisted of patients in 
whom surgery has been performed in the University Medical Centres as well as in referring peripheral hospitals. All DTC patients with MO disease who had undergone (near-) total thyroidectomy followed by I-131 ablation were included. Tumour staging was scored according to the criteria of the fifth TNM Atlas. In all patients with N1 disease, a neck dissection was performed in addition to the near-total neck dissection and prior to ablation. So, in patients with N1 disease, this stage was established before ablation. All patients with M1 disease were excluded to avoid a bias. M1 disease as exclusion criterion was based on postablation whole-body scans with high I-131 doses and radiological examinations, such as CT scanning and/or chest-X-rays, in patients with $\mathrm{T} 4$ and/or N1 disease. Finally, additional inclusion criteria were: a) ablation has been performed in accordance with either protocol and b) 6-12 months after ablation patients returned for follow-up studying consisting of Tg-off measurements and/or I-131 whole-body scintigraphy.

\section{Uptake-related ablation protocol}

This treatment strategy was used at the LUMC until June 2002. All patients had undergone (near-) total thyroidectomy, followed by I-131 ablation 4-6 weeks after surgery. During this interval, no suppressive treatment with L-thyroxine was initiated. Furthermore, low-iodine diets were prescribed to optimize the therapeutic outcome $(11,12)$. The 24 -h pre-treatment radioiodine uptake percentage in the neck region was measured using standard techniques: $40 \mathrm{MBq}$ of I-131 was given orally, followed by planar scintigraphy of the neck region $24 \mathrm{~h}$ later. A standard of $40 \mathrm{MBq}$ I-131 that was calibrated on the day of admission and measured in a neck phantom after $24 \mathrm{~h}$ was used as a reference. An uptake of less than $5 \%$, between 5 and $10 \%$, and more than $10 \%$ was followed by 2800,1850 and $1110 \mathrm{MBq}$ of I-131 in one administration respectively. The rationale of this quantitative approach was to avoid unnecessary exposure (13) and local side effects from radioiodine $(14,15)$. In this regimen, no adjustments were made in the case of cervical lymph node metastases.

\section{Fixed-dose ablation protocol}

This strategy was used in the UMCU from 1990 onwards. All patients had undergone (near-) total thyroidectomy, followed by I-131 ablation 4-6 weeks after surgery. A standard activity of $3700 \mathrm{MBq}$ was administrated in cases without any (known) metastases. In case of pre- or peri-operatively detected lymph node involvement or T4 tumour stage, an activity of $5550 \mathrm{MBq}$ was given. Between surgery and ablation patients did not receive L-thyroxine supplementation, and they had been instructed to keep a low-iodine diet for approximately 1 week $(11,12)$. No pre-ablative diagnostic scintigraphy was performed.

\section{Follow-up strategy}

Between 6 and 12 months after I-131 ablation, patients were evaluated by the measurement of Tg-off values, i.e. $\mathrm{Tg}$ values under thyrotrophin (TSH) stimulation. For this purpose, hormonal supplementation was withdrawn for 4 weeks, whereas in a minority of the UMCU cohort recombinant human TSH was applied. In the UMCU study group, all patients underwent I-131 whole body scintigraphy (WBS), whereas in the LUMC cohort I-131 WBS was performed in case of $\mathrm{Tg}<1 \mu \mathrm{g} / \mathrm{l}$. In both hospitals, scintigraphy was performed at least 3 days after administration of I-131. In case of increased $\mathrm{Tg}$ levels or an abnormal WBS, additional treatment was given followed by WBS within 7 days after the administration of a therapeutic dose. Follow-up results 6-12 months after this treatment were not studied. Despite the increased use of ultrasonography in the follow-up of DTC patients, it was not routinely used in this study.

\section{Laboratory analysis}

From 1990, in both hospitals, various kits were used for the measurement of $\mathrm{Tg}$ and $\mathrm{Tg}$ antibodies. Test results for $\mathrm{Tg}$ cannot be considered reliable in the presence of $\mathrm{Tg}$ antibodies $(16,17)$. As all assays were IRMA assays, the presence of $\mathrm{Tg}$ antibodies would have resulted in unreliable $\mathrm{Tg}$ values. Therefore, $\mathrm{Tg}$ values were excluded from analysis in the presence of measurable $\mathrm{Tg}$ antibodies and a corresponding $\mathrm{Tg}$ value below the cut-off level. As results of Tg measurements are not interchangeable between kits (18), $\mathrm{Tg}$ values in any patient were considered undetectable if they were below the lower detection limit of the kit used (i.e. cut-off level). Until 1997, serum Tg was measured using an IRMA, the Dynotest TG (Brahms Diagnostica GmBH, Berlin, Germany), with a detection limit of $1 \mu \mathrm{g} / \mathrm{l}$. From 1997 onwards, the Dynotest TG-s (Brahms Diagnostica $\mathrm{GmBH})$ was used, with a detection limit of $0.5 \mu \mathrm{g} / \mathrm{l}$. Recurrent disease, however, was defined as $\mathrm{Tg}$ levels $>1 \mu \mathrm{g} / \mathrm{l}$. TSH levels were measured by means of an immunofluorometric assay with the Delfia (Wallac, Turku, Finland) until 1997. Thereafter, an immunoluminometric assay was used with the Elecsys (Boehringer Mannheim). Serum Tg-antibodies were determined by the Ab-HTGK-3 IRMA test (DiaSorin Biomedics, Saluggia (VC) Italy).

\section{Criteria for successful ablation}

Ablation was considered successful if 6-12 months after ablation when patients fulfilled the following criteria: 
Tg-off values below the cut-off level of the assay used and negative I-131 whole-body scintigraphy.

\section{Statistical analysis}

For statistical analysis, we used SPSS version 12.0.1 for Windows (SPSS Inc., Chicago, Illinois, USA). The quantitative data (continuous parameters) were analysed using the Mann-Whitney $U$ test. For categorical data, the $\chi^{2}$-test was used. A statistically significant difference was considered when $P<0.05$.

\section{Results}

\section{Study population}

In this study, a total of 359 patients were included. According to the uptake-related and fixed-dose ablation protocols, 153 and 206 patients were treated respectively. In Table 1, the patient characteristics and results of tests for differences between the two groups are displayed. Papillary microcarcinomas were not observed in the groups studied. In the uptake-related protocol, $20 \%$ of patients were treated with $1110 \mathrm{MBq}$ I-131, $19 \%$ with $1850 \mathrm{MBq}$ and $61 \%$ with $2800 \mathrm{MBq}$. The mean 24-h I-131 uptake value in this group was $6.86 \%$ (range: $0.03-12.0 \%$ ). In the fixed-dose protocol, $69 \%$ of patients were treated with $3700 \mathrm{MBq}$ I-131 and 31\% with $5550 \mathrm{MBq}$. In this group, 24-h uptake values were not measured.

According to the evaluation with I-131 WBS as single tool (see Table 2), 89 out of 153 (58\%) patients had no iodine uptake in the neck at the first follow-up

Table 1 Differences in population characteristics for the ablation protocols studied.

\begin{tabular}{llll}
\hline & $\begin{array}{c}\text { Uptake } \\
\text { related }\end{array}$ & $\begin{array}{l}\text { Fixed } \\
\text { dose }\end{array}$ & $\boldsymbol{P}$ value \\
\hline Total number of patients & 153 & 206 & \\
Mean age (years) (range) & $\begin{array}{c}42.6 \\
(15-87)\end{array}$ & $\begin{array}{c}43.1 \\
(19-82.0)\end{array}$ & 0.675 \\
& & & 0.07 \\
Gender & $33(22)$ & $62(30)$ & \\
Male patients (\%) & $120(78)$ & $144(70)$ & \\
Female patients (\%) & & & 0.294 \\
Histology & $123(80)$ & $156(76)$ & \\
Papillary (\%) & $30(20)$ & $50(24)$ & \\
Follicular (\%) & $6.86 \%$ & NA & \\
24-h I-131-uptake (\%) & $(0.03-12)$ & & 0.008 \\
N-stage & $125(82)$ & $140(68)$ & \\
N0 (\%) & $27(18)$ & $66(32)$ & \\
N1 (\%) & $1(<1)$ & $0(0)$ & 0.005 \\
NX (\%) & $131(86)$ & $194(94)$ & \\
T-stage & $22(14)$ & $12(6)$ & \\
T1-3 (\%) & &
\end{tabular}

$\mathrm{N}$-stage, lymph node stage (NO, without clinically lymph node metastases; $\mathrm{N} 1$, with histopathologically proven lymph node metastases); T-stage, primary tumour stage. scintigraphy in the uptake-related ablation protocol. In 174 out of 206 patients ( $84 \%$ ) treated according to the fixed-dose ablation, scintigraphy did not show radioiodine uptake in the neck. This difference was statistically significant $(P<0.001)$. The scintigraphic ablation results in various subgroups as well as the differences between the protocols are displayed in Table 2, demonstrating significant differences between the different protocols in all subgroups with the exception of $\mathrm{T} 4$ tumour stages.

Regarding the combination of I-131 WBS and Tg measurement as evaluation tools, it was decided to exclude patients with $\mathrm{Tg}$ antibodies and a negative diagnostic I-131 WBS to avoid a bias leaving 338 patients available for analysis. Of those treated according to the uptake-related ablation protocol, 60 out of $139(43 \%)$ patients were successfully treated. For patients treated according to the fixed-dose ablation protocol, 111 out of 199 (56\%) patients had successful ablation. Again, this difference was statistically significant $(P=0.022)$. The results of ablation in various subgroups and tests for differences between the protocols are also displayed in Table 2. We found significant differences between the protocols for almost all subgroups defined. However, differences were not statistically significant for patients with papillary thyroid cancer $(P=0.23)$ and for patients with $\mathrm{N} 1$ $(P=0.08)$ and/or T4 $(P=0.581)$ disease. In addition, 10 patients in the uptake-related dose group and 9 patients in the fixed-dose group had T4N1 disease $(P=0.006)$. In both subgroups, only one patient had a successful ablation $(P=0.937)$ revealing a high failure rate in these tumour stages. All patients with radioiodine uptake in the neck and/or elevated $\mathrm{Tg}$ levels 6-12 months after ablation, the so-called failures, underwent subsequent treatment with a high therapeutic I-131 dose. The results of the post-therapy scans were not part of the present study, but all patients with uptake on the diagnostic follow-up WBS also demonstrated uptake on the post-therapy scan. The post-therapy scans of patients with increased Tg levels were not evaluated.

\section{Discussion}

In the present study, two fundamentally different ablation strategies were compared. The rationale of the quantitative uptake-related protocol was to avoid unnecessary exposure (13) and minimize local radioiodine side effects $(14,15)$, whereas the fixed-dose ablation protocol was designed to maximize the chance of successful ablation after one administration of I-131. The present study showed that the fixed-dose ablation protocol has a significantly higher rate of successful ablation compared with the uptake-related ablation strategy. However, of all subgroups, the fixed-dose ablation protocol failed to show a significant advantage 
Table 2 Successful ablation results in the entire population and various subgroups according to I-131 WBS and I-131 WBS with thyroglobulin $(\mathrm{Tg})$ measurements.

\begin{tabular}{|c|c|c|c|c|c|c|}
\hline \multirow[b]{2}{*}{ Group } & \multicolumn{3}{|c|}{ I-131 Whole-body scan } & \multicolumn{3}{|c|}{ I-131 Whole-body scan and Tg } \\
\hline & $\begin{array}{l}\text { Uptake related } \\
(n=153)(\%)\end{array}$ & $\begin{array}{l}\text { Fixed-dose } \\
(n=206)(\%)\end{array}$ & $P$ value & $\begin{array}{l}\text { Uptake related } \\
(n=139)(\%)\end{array}$ & $\begin{array}{l}\text { Fixed-dose } \\
(n=199)(\%)\end{array}$ & $P$ value \\
\hline All patients & $89(58)$ & $174(84)$ & $<0.001$ & $60(43)$ & $111(56)$ & 0.022 \\
\hline Ptc & 73 (59) & $133(85)$ & $<0.001$ & $47(43)$ & $75(50)$ & 0.230 \\
\hline Ftc & 16 (53) & 41 (82) & 0.006 & $13(45)$ & $36(72)$ & 0.016 \\
\hline No & $76(61)$ & 119 (85) & $<0.001$ & $55(48)$ & 86 (63) & 0.017 \\
\hline N1 & $12(44)$ & 55 (83) & $<0.001$ & $5(20)$ & $25(40)$ & 0.080 \\
\hline T1-3N0 & 70 (62) & $117(85)$ & $<0.001$ & $53(51)$ & $86(65)$ & 0.034 \\
\hline $\mathrm{T} 4$ & $10(45)$ & $12(75)$ & 0.236 & $3(15)$ & $1(8)$ & 0.581 \\
\hline
\end{tabular}

Twenty-one patients with positive Tg antibodies were excluded in the evaluation using I-131 WBS and Tg measurements. Ptc, papillary thyroid carcinoma; Ftc, follicular thyroid carcinoma; $\mathrm{N}$, lymph node stage; $\mathrm{T}$, tumour stage.

in patients with extra thyroidal invasion of the primary tumour (T4 tumours) and/or lymph node involvement (N1 stage). In addition, the results also demonstrate the low sensitivity of I-131 WBS in the follow-up of thyroid cancer patients compared with the Tg measurements. This finding is in agreement with the recommendation presented in the European guidelines indicating that for the control of successful ablation in low-risk patients $\mathrm{Tg}$ measurements are the first step in the follow-up and WBS is not recommended anymore.

Several studies have shown that higher administered I-131 activities lead to higher ablation rates, although estimations of the optimal activity vary between authors. A randomized trial reported by Bal et al. (19) revealed no differences in the rates of remnant ablation for activities over $925 \mathrm{MBq}(25 \mathrm{mCi})$. With successful ablation defined as a follow-up scintigram of the neck with less than $0.2 \%$ uptake and $\mathrm{Tg}$ less than or equal to $10 \mu \mathrm{g} / \mathrm{l}$, they achieved in $81.6 \%$ of the cases successful ablation with activities of $925 \mathrm{MBq}$ or more after one administration. The absence of activity-related differences in success rates of ablation in the study by Bal et al. is in sharp contrast with the differences encountered in the present study between those treated with a lower activity (with a minimum of $1110 \mathrm{MBq}$ ) and a higher activity (minimum $3700 \mathrm{MBq}$ ). The findings of Bal et al. are also in contrast with the results described in their earlier study (5), in which an activity of $1850 \mathrm{MBq}(50 \mathrm{mCi})$ or more performed significantly better than an activity of $1110 \mathrm{MBq}$.

Also in contrast to our results are the findings of Johansen et al. (4), who found a success rate of $81 \%$ after one administration of $1073 \mathrm{MBq}$ and $84 \%$ after the first administration for those receiving $3700 \mathrm{MBq}$. However, they analyzed only 63 patients in total, whereas their reported success rates have been based on scintigrams performed 3-4 months after ablation. They also reported that the elevated $\mathrm{Tg}$ values in the ablated subjects were not statistically significant. However, their lower detection level for $\mathrm{Tg}$ was $5 \mu \mathrm{g} / \mathrm{l}$, which is higher than our cut-off levels $(0.2-1.0 \mu \mathrm{g} / \mathrm{l})$. The comparable ablation results of 1073 and
$3700 \mathrm{MBq}$ could be caused by less sensitive follow-up (short follow-up period after ablation and higher cut-off levels for $\mathrm{Tg}$ ).

An interesting comparison can be made with the uptake-related protocol published by Zidan et al. (20), who reported results of an uptake-related protocol with used activities varying from $3145 \mathrm{MBq}(85 \mathrm{mCi})$ for the patients with lowest uptake to $1110 \mathrm{MBq}(30 \mathrm{mCi})$ for those with the highest uptake. Despite the fact that their definition of a successful ablation was based solely on a diagnostic I-131 WBS, they reported a higher overall success rate of $94 \%$. However, compared with the uptakerelated protocol as described in the present study, Zidan et al. used higher I-131 activities (approximately $1100 \mathrm{MBq}$ more) for uptake values between 6 and 15\%.

In two recently published articles, systematic metaanalyses were presented on the I-131 activity for remnant ablation in patients with DTC. In the analysis by Hackshaw et al. (21), 41 case notes, 12 prospective cohorts and 6 randomized trials were used to compare the outcome in patients treated with $30 \mathrm{mCi}$ with those treated with $100 \mathrm{mCi}$. The pooled ablation success rates in the observational studies were $10 \%$ lower using $30 \mathrm{mCi}$ compared with the higher dose. The metaanalysis of the randomized trials revealed equivocal results. Despite these findings and because of the lack of randomized trials, these authors concluded that it is not possible to reliably determine whether ablation success rates using the low activity are similar to those using the high activity. This statement was in contrast with the analysis presented by Doi et al. (22). In line with their previous report, they clearly stated that the available data continue to favour higher doses of radioiodine (ranging from 2775 to $3700 \mathrm{MBq}$ ) for remnant ablation, especially after near-total thyroidectomy. The use of high doses in these patients results in about one-third less risk of non-ablation than low doses. Our data support the use of higher doses in thyroid cancer.

Regarding the results in the present study and the data published in the literature, it is highly important to stress the difference between the I-131 activity administered and 
the absorbed dose of radiation in thyroid tissue. The absorbed dose causes the ablation effect. This dose depends on several factors, such as uptake of I-131 and retention time in the remnants, the mass of the thyroid remnant, different TSH levels, the initial activity given and patient's preparation. Despite the fact that a standard amount of radioactivity is used in the present study protocols, it may still result in different ablation doses.

In both academic hospitals, the aim of surgery is to do an optimal resection of malignant tissue, which is a combination of a (near-) total thyroidectomy combined with a neck dissection in case of lymph node metastases. In T4 tumours, there seems to be, however, a high change on residual malignant cells in the thyroid remnants. In addition, a comparable phenomenon has been described for $\mathrm{N} 1$ disease, in which even after a modified neck dissection micrometastases in left lymph nodes cannot be excluded. In the literature, it has been described that thyroid carcinoma cells take up and process iodine less efficiently than normal thyroid cells due to a lower expression of the sodium-iodine symporter (23-25). In a recently published study on prognostic parameters in thyroid cancer, both N1 and T4 tumour stages significantly correlated with a high chance on local tumour recurrences (26). Consequently, minimal residual disease could be a thorough explanation for the fact that our study did not show a statistically significant difference in ablation results between the two protocols in case of $\mathrm{T} 4$ and/or N1 tumours. Even a mean activity of I-131 up to $5000 \mathrm{MBq}$ as used in the fixed ablation dose, which is twice as much as applied in the uptake-related strategy, fails to achieve a complete response in 60 and $92 \%$ of the patients with $\mathrm{N} 1$ and $\mathrm{T} 4$ tumour stage respectively. In addition, in only 1 out of 10 and 9 patients with T4N1 disease in the uptake-related and fixed-dose strategies respectively, success was achieved. These findings are in agreement with data published by Rosaria et al. (27). They studied 274 patients with DTC and found a clear relationship between ablation failures and the presence of metastases and tumours larger than $4 \mathrm{~cm}$ in diameter. However, also thyroid remnants with an uptake $>5 \%$ resulted in a higher failure rate.

It has to be realized that two factors could have influenced the treatment results in the present study. First, the two centres used different follow-up strategies. An endocrinologist of the LUMC performed the clinical follow-up of patients who underwent ablation in the LUMC. The clinical follow-up of a part of the UMCU patients is in their own (referring) hospitals by means of I-131 diagnostic scintigraphy and $\mathrm{Tg}$ measurements. This subgroup of UMCU patients only returns to the UMCU if additional radioiodine treatment is required (in case of unsuccessful ablation). This effectively may create a bias, as a number of patients with a successful ablation cannot be included in the present study. In this respect however, the missing data would have further increased the overall success rate in the fixed-dose group compared with the uptake-related protocol. Second, it has been shown that relatively low diagnostic activities of I-131 may lead to impaired ability of remnant thyroid tissue to concentrate the subsequent ablative dose of I-131 (so-called stunning effect) and thereby reduce therapeutic efficacy (28-31). Although this phenomenon has been acknowledged for some time, the precise time interval and I-131 activity after which it occurs is still a subject to discussion. If an applied activity of $40 \mathrm{MBq}$ causes a lower concentration of I-131 in the thyroid remnant, part of the effect seen in this study could have been attributed to the stunning effect with a probable bias against the uptake-related protocol. However, data on stunning effects caused by such low doses have not been reported yet.

In the present study, we evaluated patients at 6-12 months after ablation, demonstrating a significant difference in short-term outcome. However, data on long-term recurrence rates are currently not available. The number of studies evaluating differences in longterm outcome in patients treated with high or low I-131 doses is scarce. Data presented by Chow et al. clearly demonstrated the influence of radioiodine after surgery regarding the 5-, 10- and 15-year local relapse rate (32). In patients not treated by radioiodine after surgery, the cumulative relapse rate after 15 years follow-up was $20.9 \%$ compared with $9.2 \%$ in patients treated with radioiodine. In this study, patients who were treated received a mean dose of $3400 \mathrm{MBq}$ at initial stage. It was shown by Verburg et al., that a successful ablation itself seems to be a highly important prognostic factor for long-term outcome (33). They found that of the patients with a successful ablation, $87 \%$ were still free of the disease after 10 years, whereas of the patients with an unsuccessful ablation, only 50\% were free of disease $(P<0.001)$. According to this finding, a higher recurrence rate may be expected during follow-up in the uptake-related protocol group compared with the group of patients treated according to the fixed-dose protocol. However, this statement is not supported by data recently published by Rosario et al. (34). They treated 82 patients with $3700 \mathrm{MBq} \mathrm{I-131}$ and 44 patients with $1100 \mathrm{MBq}$. At the end of a 5-year followup period, the recurrence rate was $3.6 \%$ in patients who had received the high dose and $3.4 \%$ in those treated with the low dose $(P=N S)$. Criteria used for patient's selection and risk factors were unfortunately not reported, which may have caused a selection bias. Therefore, more randomized trials are required to assess the short- and long-term outcome in relation to the ablation dose used in DTC ablation.

\section{Conclusion}

The fixed-dose ablation protocol, using relatively higher I-131 activities, is generally more effective in thyroid remnant ablation than a 24-h I-131 uptake-related 
ablation protocol that uses relatively low activities. This difference, however, is not observed in patients with $\mathrm{T} 4$ and/or N1 tumour stages, which can be clarified by the presence of minimal residual malignant disease. The present study addressed the issue of ablation efficacy as judged by scintigraphy and $\mathrm{Tg}$ measurements after 6-12 months, whereas long-term outcome was not evaluated. Therefore, follow-up studies are necessary to solve whether this lack of difference between both algorithms results in poorer long-term outcome.

\section{References}

1 Utiger RD. Follow-up of patients with thyroid carcinoma. New England Journal of Medicine 1997337 928-930.

2 Schlumberger MJ. Papillary and follicular thyroid carcinoma. New England Journal of Medicine 1998338 297-306.

3 Creutzig H. High or low dose radioiodine ablation of thyroid remnants? European Journal of Nuclear Medicine 198712 500-502.

4 Johansen K, Woodhouse NJ \& Odugbesan O. Comparison of $1073 \mathrm{MBq}$ and $3700 \mathrm{MBq}$ iodine-131 in postoperative ablation of residual thyroid tissue in patients with differentiated thyroid cancer. Journal of Nuclear Medicine 199132 252-254.

5 Bal C, Padhy AK, Jana S, Pant GS \& Basu AK. Prospective randomized clinical trial to evaluate the optimal dose of $131 \mathrm{I}$ for remnant ablation in patients with differentiated thyroid carcinoma. Cancer 199677 2574-2580.

6 Siddiqui AR, Edmondson J, Wellman HN, Hamaker RC, Lingeman RE, Park HM \& Johnston CC. Feasibility of low doses of I-131 for thyroid ablation in postsurgical patients with thyroid carcinoma. Clinical Nuclear Medicine 19816 158-161.

7 Doi SA \& Woodhouse NJ. Ablation of the thyroid remnant and $131 \mathrm{I}$ dose in differentiated thyroid cancer. Clinical Endocrinology 200052 765-773.

8 Verkooijen RB, Stokkel MP, Smit JW \& Pauwels EK. Radioiodine131 in differentiated thyroid cancer: a retrospective analysis of an uptake-related ablation strategy. European Journal of Nuclear Medicine and Molecular Imaging 200431 499-506.

9 de Klerk JM, de Keizer B, Zelissen PM, Lips CM \& Koppeschaar HP. Fixed dosage of 131I for remnant ablation in patients with differentiated thyroid carcinoma without pre-ablative diagnostic 131 I scintigraphy. Nuclear Medicine Communications 200021 529-532.

10 Verburg FA, de Keizer B, Lips CJ, Zelissen PM \& de Klerk JM. Prognostic significance of successful ablation with radioiodine of differentiated thyroid cancer patients. European Journal of Endocrinology $200515233-37$.

11 Pluijmen MJ, Eustatia-Rutten C, Goslings BM, Stokkel MP, Arias AM, Diamant M, Romijn JA \& Smit JW. Effects of low-iodide diet on postsurgical radioiodide ablation therapy in patients with differentiated thyroid carcinoma. Clinical Endocrinology $2003 \mathbf{5 8}$ 428-435.

12 Goslings BM. Proceedings: effect of a low iodine diet on 131-I therapy in follicular thyroid carcinomata. Journal of Endocrinology $19756430 P$.

13 Maxon HR III, Englaro EE, Thomas SR, Hertzberg VS, Hinnefeld JD, Chen LS, Smith H, Cummings D \& Aden MD. Radioiodine-131 therapy for well-differentiated thyroid cancer - a quantitative radiation dosimetric approach: outcome and validation in 85 patients. Journal of Nuclear Medicine 199233 1132-1136.

14 Alexander C, Bader JB, Schaefer A, Finke C \& Kirsch CM. Intermediate and long-term side effects of high-dose radioiodine therapy for thyroid carcinoma. Journal of Nuclear Medicine 1998 39 1551-1554.
15 Burmeister LA, du Cret RP \& Mariash CN. Local reactions to radioiodine in the treatment of thyroid cancer. American Journal of Medicine $199190217-222$.

16 Spencer CA, Takeuchi M, Kazarosyan M, Wang CC, Guttler RB, Singer PA, Fatemi S, LoPresti JS \& Nicoloff JT. Serum thyroglobulin autoantibodies: prevalence, influence on serum thyroglobulin measurement, and prognostic significance in patients with differentiated thyroid carcinoma. Journal of Clinical Endocrinology and Metabolism $1998 \mathbf{8 3} 1121-1127$.

17 Mariotti S, Barbesino G, Caturegli P, Marinó M, Manetti L, Pacini F, Centoni R \& Pinchera A. Assay of thyroglobulin in serum with thyroglobulin autoantibodies: an unobtainable goal? Journal of Clinical Endocrinology and Metabolism $1995 \mathbf{8 0}$ 468-472.

18 Spencer CA, Bergoglio LM, Kazarosyan M, Fatemi S \& LoPresti JS. Clinical impact of thyroglobulin ( $\mathrm{Tg}$ ) and $\mathrm{Tg}$ autoantibody method differences on the management of patients with differentiated thyroid carcinomas. Journal of Clinical Endocrinology and Metabolism 200590 5566-5575.

19 Bal CS, Kumar Ajay \& Pant GS. Radioiodine dose for remnant ablation in differentiated thyroid carcinoma: a randomized clinical trial in 509 patients. Journal of Clinical Endocrinology and Metabolism 200489 1666-1673.

20 Zidan J, Hefer E, Iosilevski G, Drumea K, Stein ME, Kuten A \& Israel O. Efficacy of 1131 ablation therapy using different doses as determined by postoperative thyroid scan uptake in patients with differentiated thyroid cancer. International Journal of Radiation Oncology, Biology, Physics 200459 1330-1336.

21 Hakshaw A, Harmer C, Mallick U, Haq M \& Franklyn JA. Review: I-131 activity for remnant ablation in patients with differentiated thyroid cancer: a systematic review. Journal of Clinical Endocrinology and Metabolism 200792 23-28.

22 Doi SAR, Woodhouse NJ, Thalib L \& Onitilo A. Ablation of the thyroid remnant and I-131 dose in differentiated thyroid cancer: a meta-analysis revisited. Clinical Medicine E Research 20075 87-90.

23 Lazar V, Bidart JM, Caillou B, Mahé C, Lacroix L, Filetti S \& Schlumberger M. Expression of the $\mathrm{Na}+/ \mathrm{I}-$ symporter gene in human thyroid tumors: a comparison study with other thyroidspecific genes. Journal of Clinical Endocrinology and Metabolism $1999843228-3234$.

24 Arturi F, Russo D, Schlumberger M, du Villard JA, Caillou B, Vigneri P, Wicker R, Chiefari E, Suarez HG \& Filetti S. Iodide symporter gene expression in human thyroid tumors. Journal of Clinical Endocrinology and Metabolism $1998 \mathbf{8 3}$ 2493-2496.

25 Gerard AC, Daumerie C, Mestdagh C, Gohy S, DeBurbure C, Costagliola S, Miot F \& Nollevaux MC. Correlation between the loss of thyroglobulin iodination and the expression of thyroidspecific proteins involved in iodine metabolism in thyroid carcinomas. Journal of Clinical Endocrinology and Metabolism $2003 \mathbf{8 8} 4977-4983$.

26 Verkooijen RB, Rietbergen D, Smit JW, Romijn JA \& Stokkel MP. A new functional parameter measured at the time of ablation that can be used to predict differentiated thyroid cancer recurrence during follow-up. European Journal of Endocrinology $200715641-47$.

27 Rosario PWS, Reis JS, Barroso AL, Rezende LL, Padrao EL \& Fagundes TA. Efficacy of low and high I131 doses for thyroid remnant ablation in patients with differentiated thyroid carcinoma based on post-operative cervical uptake. Nuclear Medicine Communications 200425 1077-1081.

28 Park HM, Perkins OW, Edmondson JW, Schnute RB \& Manatunga A. Influence of diagnostic radioiodines on the uptake of ablative dose of iodine-131. Thyroid 1994 4 49-54.

29 Muratet JP, Daver A, Minier JF \& Larra F. Influence of scanning doses of iodine-131 on subsequent first ablative treatment outcome in patients operated on for differentiated thyroid carcinoma. Journal of Nuclear Medicine 199839 1546-1550. 
30 Leger FA, Izembart M, Dagousset F, Barritault L, Baillet G, Chevalier A \& Clerc J. Decreased uptake of therapeutic doses of iodine-131 after 185-MBq iodine-131 diagnostic imaging for thyroid remnants in differentiated thyroid carcinoma. European Journal of Nuclear Medicine 199825 242-246.

31 Lassmann M, Luster M, Hanscheid H \& Reiners C. Impact of 131 I diagnostic activities on the biokinetics of thyroid remnants. Journal of Nuclear Medicine 200445 619-625.

32 Chow SM, Law SC, Mendenhall WM, Au SK, Chan PT, Leung TW, Tong CC, Wong IS \& Lau WH. Papillary thyroid carcinoma: prognostic factors and the role of radioiodine and external radiotherapy. International Journal of Radiation Oncology, Biology, Physics 200252 784-795.
33 Verburg FA, de Keizer B, Lips CJM, Zelissen PMJ \& de Klerk JHM. Prognostic significance of successful ablation with radioiodine of differentiated thyroid cancer patients. European Journal of Endocrinology 2005152 33-37.

34 Rosario PWS, Purish S, Vasconcelos FPJ, Padrao EL, Rezende LL \& Barroso AL. Long-term recurrence of thyroid cancer after thryoid remnant ablation with 1.1 and $3.7 \mathrm{GBq}$ radioiodine. Nuclear Medicine Communications 200728 507-508.

Received 2 June 2008

Accepted 6 June 2008 